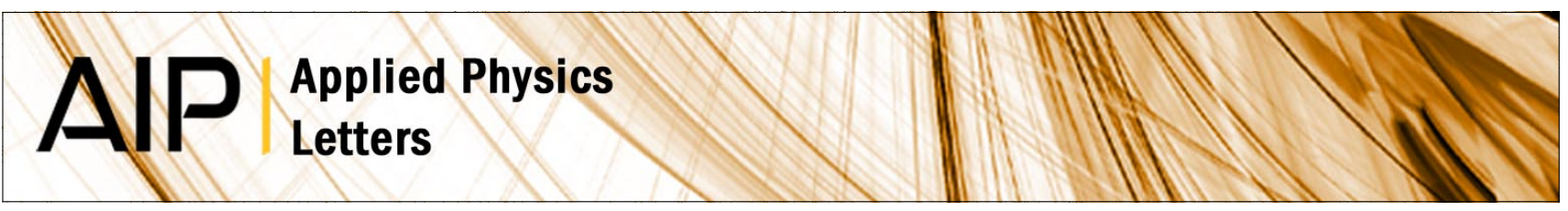

\title{
What is the useful energy of a photon?
}

Tom Markvart and Gottfried H. Bauer

Citation: Appl. Phys. Lett. 101, 193901 (2012); doi: 10.1063/1.4766177

View online: http://dx.doi.org/10.1063/1.4766177

View Table of Contents: http://apl.aip.org/resource/1/APPLAB/v101/i19

Published by the American Institute of Physics.

\section{Related Articles}

Highly efficient indium tin oxide-free organic photovoltaics using inkjet-printed silver nanoparticle current collecting grids

Appl. Phys. Lett. 101, 193302 (2012)

Highly efficient indium tin oxide-free organic photovoltaics using inkjet-printed silver nanoparticle current collecting grids

APL: Org. Electron. Photonics 5, 242 (2012)

Tunable photovoltaic effect and solar cell performance of self-doped perovskite $\mathrm{SrTiO} 3$

AlP Advances 2, 042131 (2012)

Modeling of downconverter based on Pr3+-Yb3+ codoped fluoride glasses to improve sc-Si solar cells efficiency AIP Advances 2, 042130 (2012)

Optical approaches to improve the photocurrent generation in $\mathrm{Cu}(\mathrm{In}, \mathrm{Ga}) \mathrm{Se} 2$ solar cells with absorber thicknesses down to $0.5 \mu \mathrm{m}$

J. Appl. Phys. 112, 094902 (2012)

\section{Additional information on Appl. Phys. Lett.}

Journal Homepage: http://apl.aip.org/

Journal Information: http://apl.aip.org/about/about_the_journal

Top downloads: http://apl.aip.org/features/most_downloaded

Information for Authors: http://apl.aip.org/authors

\section{ADVERTISEMENT}
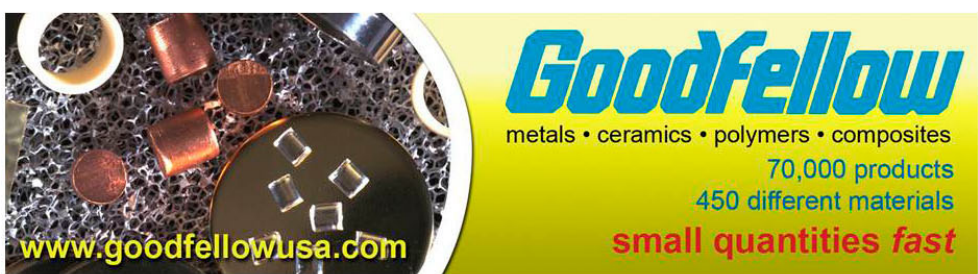


\title{
What is the useful energy of a photon?
}

\author{
Tom Markvart ${ }^{1, \text { a) }}$ and Gottfried H. Bauer ${ }^{2, b)}$ \\ ${ }^{1}$ School of Engineering Sciences, University of Southampton, Southampton SO17 1BJ, United Kingdom \\ ${ }^{2}$ Division of Semiconductor Physics, Institute of Physics, CvO University, D-26111 Oldenburg, Germany
}

(Received 12 June 2012; accepted 22 October 2012; published online 7 November 2012)

The fundamental upper bound on the efficiency of photovoltaic conversion continues to attract interest of the research community. By considering the conversion efficiency of a monochromatic photon gas at constant pressure, we show that this limit is equal to the availability (or exergy), as defined in textbooks on classical thermodynamics. The application of this result to the full spectrum of black-body radiation yields the Petela-Press-Landsberg efficiency. Generalization to include entropy generation on account of the kinetic nature of the conversion process, by drawing a parallel with the efficiency of an infinite tandem converter, yields a theoretical efficiency limit of 85.2\%. @ 2012 American Institute of Physics. [http://dx.doi.org/10.1063/1.4766177]

Much research is currently under way with the aim of increasing the conversion efficiency beyond the ShockleyQueisser single junction limit ${ }^{1}$ but the maximum efficiency that can be achieved remains the subject of debate. Frequently discussed is an infinite tandem device where each spectral component is converted with optimum efficiency. ${ }^{2}$ This can be compared with the somewhat higher efficiency determined by Landsberg and Mallinson ${ }^{3}$ using methods of thermodynamics (see also Refs. 4 and 5). We shall show that these two efficiency limits are intimately related and, if viewed on a rigorous footing, provide a glimpse on different aspects of the conversion process. When combined, a new thermodynamic efficiency limit is obtained where losses are included transparently through unavoidable entropy generation terms. Unlike all theoretical limits obtained so far, the present limit describes a conversion process with a photon gas taking place under constant pressure rather than at constant volume.

The photovoltaic conversion process consists of absorption and charge separation (Fig. 1). At open circuit in the absence of nonradiative processes, all photons which are absorbed in the cell will be re-emitted. We have shown previously ${ }^{7}$ that, for identical étendue of the incident and emitted beam, no entropy is generated and the absorber would be in thermal equilibrium with the Sun, at a temperature $T_{S} \cong 6000 \mathrm{~K}$ of the solar photosphere.

In a working solar cell, photons are removed from the absorber for charge separation. We consider here an alternative picture that photons simply carry out work-for example, mechanical work or work of electrons against electrostatic forces. The conversion of a photon into maximum value of this work can then be equated to $q V_{o c}$, where $V_{o c}$ is the open circuit voltage of the solar cell and $q$ is the elementary charge. The conversion of photon energy into work is subject to the laws of thermodynamics and, as we will show presently, the amount of work cannot be simply be equated to the usual expression $h \nu$, where $\nu$ is the photon frequency and $h$ is the Planck constant.

As part of the conversion process, the absorber energy is indeed reduced by $h \nu$. However, the photon also carries

\footnotetext{
a)Email: tm3@soton.ac.uk.

b)Email: g.h.bauer@uni-oldenburg.de.
}

entropy ( $s_{v}$, say) and the absorber entropy is, therefore, reduced by $s_{v}$ when a photon is removed. The absorber exchanges energy with a reservoir at temperature $T_{o}$ ( $\cong 300 \mathrm{~K}$ ) of the medium surrounding the solar cell. The combined system represented by the absorber and the reservoir can be viewed as isolated and, by the second law of thermodynamics, its entropy cannot increase. As a result, the entropy of the reservoir has to increase by an amount $s_{\nu}^{\prime}$ which cannot be smaller than $s_{v}$. In the familiar terminology for a heat engine, the absorber rejects heat of magnitude $T_{o} s_{\nu}^{\prime}$.

A photon removal from the absorber has yet another consequence. If this process is to be reversible, the volume per photon $\% / \delta \mathrm{n}_{\nu}(T)$ has to remain constant to ensure that the absorber remains at a constant temperature. Here,

$$
\delta n_{\nu}(T)=\vartheta \frac{8 \pi \nu^{2}}{c^{3}} \rho_{\nu}(T) \delta \nu,
$$

is the number of photons in the frequency interval $v \rightarrow v+\delta v$ in three dimensions, and

$$
\rho_{\nu}(T)=\frac{1}{e^{\frac{h \nu}{k^{T}}}-1},
$$

is the usual occupation probability of a photon state at frequency $v$. In such a process, the total volume of the photon gas decreases, and the ambient black body radiation at frequency $v$ with pressure $\delta p_{\nu}\left(T_{o}\right)$ has to expand, and performs work ${ }^{0} \delta p_{\nu}\left(T_{o}\right) / \delta n_{v}\left(T_{S}\right)$ on the absorber.

The essence of this argument is well known to any mechanical engineer who wishes to determine the maximum amount of work that can be carried out by an object which is cooled from temperature $T_{S}$ to the ambient temperature $T_{o}{ }^{6}{ }^{6}$ Here, we have applied a similar philosophy to determine the work that can be carried out by a monochromatic photon originating from a thermal source such as the Sun.

Thus, the maximum amount of work $w_{v}$ that can be carried out by the photon of energy $h v$ in the frequency interval $\delta v$ is equal to the appropriate availability (or exergy)

$$
w_{v}=h_{\nu}-T_{o} s_{v}-w_{o v},
$$




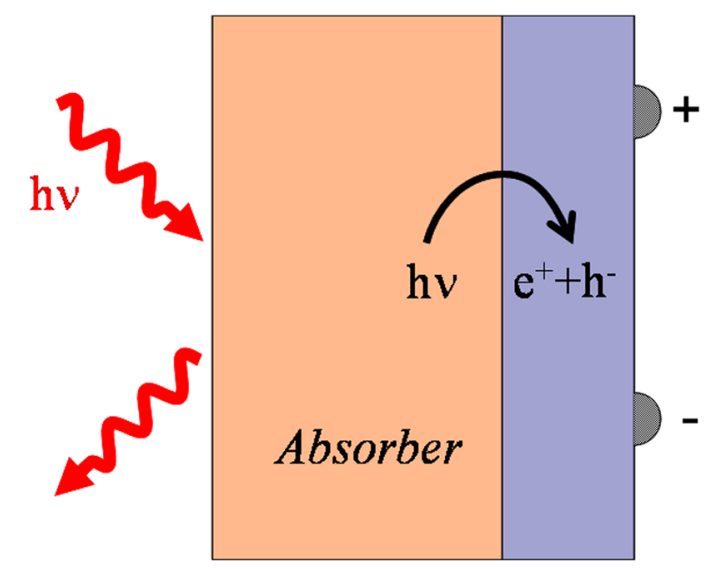

FIG. 1. A schematic depiction of photovoltaic conversion.

where

$$
w_{o v}=-\gamma \frac{\delta p_{v}\left(T_{o}\right)}{\delta n_{v}\left(T_{S}\right)}=\frac{k_{B} T_{o}}{\rho_{v}\left(T_{S}\right)} \ln \left\{\frac{1+\rho_{v}\left(T_{S}\right)}{1+\rho_{v}\left(T_{o}\right)}\right\} .
$$

The term $w_{o v}$ represents the work carried out by the ambient black-body radiation at temperature $T_{o}$, which is additional to the analysis of Ref. 7. In Eq. (4), we noted that the pressure of the monochromatic radiation is equal to

$$
\delta p_{v}(T)=\frac{8 \pi v^{2}}{c^{3}} k_{B} T \ln \left\{1+\rho_{v}(T)\right\} \delta v,
$$

which follows from the representation with the partition function of canonical ensembles and in alignment with standard texts on black-body radiation (see, for example, Ref. 6). We also used the expression for the photon entropy

$$
\begin{aligned}
s_{v} & =s_{v}(T s)=\frac{1}{T_{S}}\left\{h v+\frac{\partial \delta p\left(T_{S}\right)}{\delta n\left(T_{S}\right)}\right\} \\
& =\frac{h v}{T_{S}}+\frac{k_{B}}{\rho_{v}(T s)} \ln \left\{1+\rho_{v}(T s)\right\},
\end{aligned}
$$

which follows from the chemical potential of the black body radiation being equal to zero.

The result (3) for monochromatic light can now be used to extend the argument to black-body radiation extending over the full frequency spectrum. To this end, we replace the $\delta$ 's by infinitesimal increments and write

$$
W=\int w_{v} d n_{v} .
$$

The integral in (7) is easily evaluated,

$$
\begin{aligned}
W= & \left(1-T_{o} / T_{S}\right)\left\{\left(\frac{8 \pi^{\Upsilon} 0}{c^{3}}\right)\left(\frac{\pi^{4} k_{B}^{4} T_{S}^{4}}{15 h^{3}}\right)\right\} \\
& -\left\{\left(\frac{8 \pi^{\Upsilon} \vartheta}{c^{3}} k_{B}\right)\left(\frac{\pi^{4} k_{B}^{4}}{45 h^{3}}\right) T_{o}\right\}\left(T_{S}^{3}-T_{o}^{3}\right) \\
= & \left(1-T_{o} / T_{S}\right) U\left(T_{S}\right)-\left(T_{S}{ }^{3}-T_{o}{ }^{3}\right) \frac{U\left(T_{o}\right)}{T_{o}{ }^{3}},
\end{aligned}
$$

with the final result

$$
W=\eta_{L} U\left(T_{S}\right)
$$

where

$$
U(T)=a^{\odot} T^{4},
$$

is the internal energy of the black body radiation, $a$ is the radiation constant and $\eta_{L}$ is the Landsberg efficiency ${ }^{3-5}$

$$
\eta_{L}=1-\frac{4}{3}\left(T_{o} / T_{S}\right)+\frac{1}{3}\left(T_{o} / T_{S}\right)^{4} .
$$

Thus, the Landsberg efficiency gives the availability of the black body radiation in terms of its internal energy, as already noted in Ref. 5. It is significant that the process it describes is reversible and thus without entropy generation. The resulting work, therefore, does not allow for any "kinetic" losses which are inherent in photovoltaic conver$\operatorname{sion}^{7}$ and corresponds to a "static head" ${ }^{8}$ or open circuit, as we shall presently elucidate in more detail.

It is tempting to bring in the kinetic element by introducing the elementary currents corresponding to photon numbers $\delta n_{v}\left(T_{S}\right)$ by $\delta j_{v}^{\ell}=q c \delta n_{v}\left(T_{S}\right) / 4^{\sigma} \%$, where $c$ is the speed of light, transforming (9) into a formula for what is apparently the output power expressed in terms of the incident (and completely absorbed) radiant energy flux density (ca/4) $T_{S}{ }^{4}$. This view, however, is incorrect as the fluxes which enter here are convective flows in the sense of irreversible thermodynamics 9 rather than true thermodynamic fluxes.

To see this more clearly, we equate the spectral availability $w_{v}$ to the maximum electrostatic energy that can be produced by a solar cell which absorbs in a narrow spectral range near frequency $v$, in other words, to the product $q V_{v}{ }^{o c}$, where a subscript $v$ has been introduced to identify individual solar cells operating at different frequencies. The power produced by an infinite multijunction set of solar cells, each separately converting a narrow spectral component would then be equal to

$$
P_{L}=\sum_{v} V_{v}{ }^{o c} \delta j_{\nu}^{\ell}
$$

In this form, rudimentary solar cell theory immediately reveals that Eq. (12) represents an overestimate as it does not include the appropriate fill factors $f f_{\nu}$, each describing the operation of an elementary solar cell at frequency $v$.

The fill factor (which cannot be expressed in a closed form) is discussed in any textbook on photovoltaics but it is of interest to pause briefly and consider this quantity from a thermodynamic viewpoint. We have shown previously ${ }^{7}$ that the fill factor represents the necessary kinetic losses, as expressed by entropy generation $\sigma_{i, \mathrm{kin}}$ on account of a finite rate of turnover of the photovoltaic "conversion engine." This term is missing in the "static" description provided by the availability argument leading to the Landsberg efficiency ${ }^{9}$ which assumes reversible operation.

The analysis so far has assumed operation at open circuit when all the absorbed photons are emitted. Suppose now that current is extracted from the solar cell. If all the absorbed photons were converted into electron-hole pairs and extracted (i.e., at short circuit), only equilibrium photons at the temperature $T_{o}$ of the low-temperature reservoir would be emitted by the solar cell. These photons have a zero 


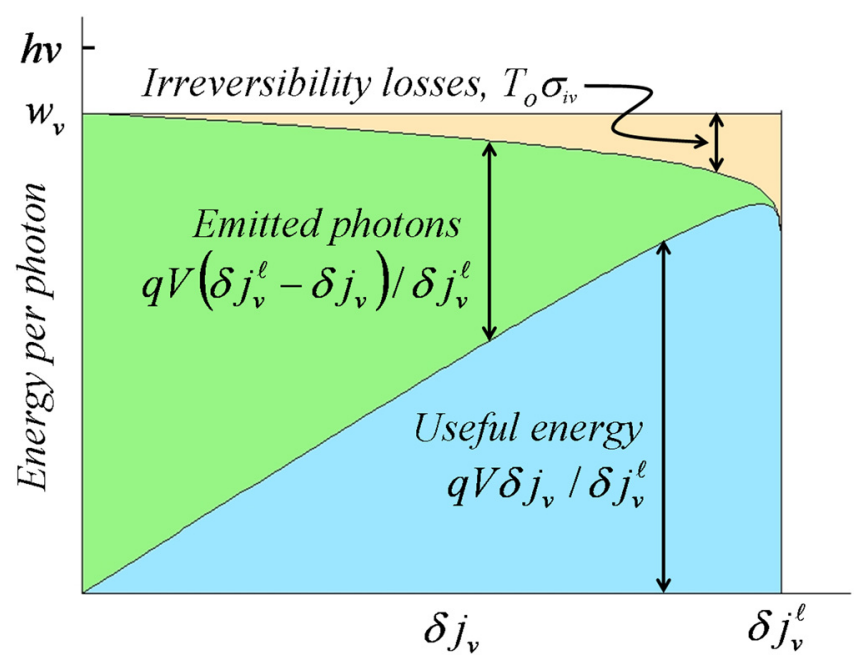

FIG. 2. The useful energy per photon of incident radiation produced by a monochromatic solar cell, showing the various losses given by Eq. (13). The curve marked useful energy will be recognized as corresponding to the power curve of an ideal solar cell but plotted here as a function of current produced by the solar cell.

chemical potential and cannot carry out useful work. In this limit, all the potential of the solar photons for work is therefore balanced by entropy generation $\sigma_{i, \mathrm{kin}}$.

In the intermediate case between these two extremes, the voltage produced by the cell is reduced from the open circuit voltage by an amount $T_{o} \sigma_{i, \mathrm{kin}} / q$ corresponding to the heat rejected to the low temperature reservoir on account of entropy generation. The power produced by the monochromatic cell at frequency $v$ is, therefore, reduced from the reversibility limit by both photon emission and entropy generation (Fig. 2), as can be seen by writing

$P_{v}=V_{v} \delta j_{v}=w_{v}\left(\delta j_{\nu}^{\ell} / q\right)-\left[T_{o} \sigma_{i, k i n}\left(\delta j_{\nu}^{\ell} / q\right)+\left(w_{\nu}-T_{o} \sigma_{i, k i n}\right) \delta \phi_{v}\right]$,

where $\delta \phi_{v}=\left(\delta j_{\nu}^{\ell}-\delta j_{\nu}\right) / q$ is the photon flux emitted by a cell producing current $\delta j_{\nu}$, and $\sigma_{i \text {,kin }}$ (which depends on $\delta j_{v}$ ) refers to the kinetic entropy generation for cell $v$. The first term on the right hand side of Eq. (13), equal to $V_{\nu}^{o c} \delta j_{\nu}^{\ell}$, is the power that would be generated without any irreversible losses. The second term represents the photon energy loss on account of entropy generation. The last term is the power carried away by photons emitted by the cell producing less than the maximum current density $\delta j_{v}^{\ell}$. The fill factor represents the losses represented by the last two terms in Eq.

(13) at the maximum power point.

Including the fill factor, the new efficiency

$$
\eta_{\max }=\frac{\int V_{v} d j_{v}}{(c a / 4) T_{S}^{4}}=\frac{\int V_{v}^{o c} \int f_{v} d j_{v}}{(c a / 4) T_{S}{ }^{4}}
$$

gives a slightly lower but more realistic and quite general upper limit to the photovoltaic conversion efficiency, where all unavoidable thermodynamic losses have been taken into account.

The efficiency (14) can be compared with the efficiency of an "infinite tandem" obtained by deVos and Pauwels. ${ }^{2} \mathrm{De}$ $\operatorname{Vos}^{10}$ has shown that the efficiency of the infinite tandem can be obtained quite simply if the open circuit voltage is described by an expression equivalent to Eq. (3) but keeping only the first, Carnot-factor like term in Eq. (3) and neglecting the term $w_{o v}$ given by Eq. (4). The correction represented by this term is not large-for most of the spectrum it can be approximated by the thermal energy $k_{B} T_{o} \cong 26 \mathrm{meV}$. Notwithstanding, it reduces the usual limit to infinite tandem efficiency from $86.8 \%$ to $85.2 \%$. This can be compared to the "open circuit" voltage limit of $93.3 \%$ represented by the Landsberg efficiency which does not make allowance for the fill factor or, equivalently, for the losses described by the two terms in square brackets in Eq. (13).

${ }^{1}$ W. Shockley and H. J. Queisser, "Detailed balance limit of efficiency of p-n junction solar cells," J. Appl. Phys. 32, 510 (1961).

${ }^{2} \mathrm{~A}$. deVos and H. Pauwels, "On the thermodynamic limit of photovoltaic energy conversion," Appl. Phys. A 25, 119, 1981.

${ }^{3}$ P. T. Landsberg and J. R. Mallinson, "Thermodynamic constraints. Effective temperatures and solar cells," in International Colloquium on Solar Electricity (C.N.E.S., Toulouse, 1976), p. 27.

${ }^{4}$ W. H. Press, "Theoretical maximum for energy from direct and diffuse sunlight," Nature 264, 734 (1976).

${ }^{5}$ R. Petela, "Energy of heat radiation," J. Heat Transfer 86, 187 (1964).

${ }^{6}$ L. D. Landau and E. M. Lifshitz, Statistical Physics (Pergamon, 1996).

${ }^{7}$ T. Markvart, "Thermodynamics of losses in photovoltaic conversion," Appl. Phys. Lett. 91, 064102 (2007); T. Markvart, "Solar cell as a heat engine: Energy-entropy analysis of photovoltaic conversion," Physica Status Solidi A 205, 2752 (2008).

${ }^{8}$ O. Kedem and S. R. Kaplan, "Degree of coupling and its relation to the efficiency of energy transduction," Trans. Faraday Soc. 61, 1897 (1965).

${ }^{9}$ See, for example, S. R. de Groot and P. Mazur, Non-Equilibrium Thermodynamics (Dover, New York, 1984).

${ }^{10} \mathrm{~A}$. deVos, "The current-voltage characteristic of the ideal two-terminal tandem solar cell," Prog. Photovoltaics 11, 237 (2003). 